\title{
(e, 2e) triple differential cross-sections for ionization beyond helium: the neon case at large energy transfer
}

\author{
A Naja ${ }^{1,2}, \mathbf{E}$ M Staicu Casagrande ${ }^{1,2}$, A Lahmam-Bennani ${ }^{1,2}$, \\ M Stevenson $^{3}$, B Lohmann ${ }^{3}$, C Dal Cappello ${ }^{4}$, K Bartschat $^{5}$, A Kheifets ${ }^{6}$, \\ I Bray $^{7}$ and D V Fursa ${ }^{7}$ \\ ${ }^{1}$ Université Paris-Sud 11, Laboratoire des Collisions Atomiques et Moléculaires (LCAM), Bât. 351, \\ 91405 Orsay Cedex, France \\ ${ }^{2}$ CNRS-LCAM (UMR 8625), Bât. 351, 91405 Orsay Cedex, France \\ ${ }^{3}$ ARC Centre of Excellence for Antimatter-Matter Studies, The University of Adelaide, Adelaide, \\ SA 5005, Australia \\ ${ }^{4}$ Université Paul Verlaine-Metz, Laboratoire de Physique Moléculaire et des Collisions, \\ ICPMB (FR 2843), Institut de Physique, 1 rue Arago, 57078 Metz Cedex 3, France \\ ${ }^{5}$ Department of Physics and Astronomy, Drake University, Des Moines, IA 50311, USA \\ ${ }^{6}$ Institute of Advanced Studies, Research School of Physical Sciences, The Australian National \\ University, Canberra ACT 0200, Australia \\ ${ }^{7}$ ARC Centre of Excellence for Antimatter-Matter Studies, Curtin University of Technology, \\ GPO Box U1987, Perth, WA 6845, Australia
}

Received 11 February 2008, in final form 11 March 2008

Published 8 April 2008

Online at stacks.iop.org/JPhysB/41/085205

\begin{abstract}
We report new coplanar (e, 2e) results for ionization of the He 1s and $\mathrm{Ne} 2 \mathrm{p}$ and $2 \mathrm{~s}$ subshells under kinematics which have remained rather unexplored to date and characterized by large energy transfer and close to minimum momentum transfer from the projectile to the target. The experimental results obtained in two laboratories are used as a sensitive test of a number of state-of-the-art available theoretical models for multi-electron atoms. The He results are in excellent agreement with convergent close-coupling predictions as well as with distorted-wave + Gamow and hybrid distorted-wave $+R$-matrix models. In the Ne case, an overall satisfactory agreement with experiments is obtained for all considered models. The importance of post-collisional interaction effects is demonstrated whereas second-order effects in the projectile-target interaction at small distances are shown to be negligibly small. Remaining discrepancies between theories and experiments are discussed.
\end{abstract}

(Some figures in this article are in colour only in the electronic version)

\section{Introduction}

The so-called (e, 2e) collisions correspond to ionizing processes induced by the impact of an incoming electron on a target, giving rise to two emerging electrons which are fully analysed in energy and momentum and detected in coincidence. The corresponding triple differential crosssection (TDCS) has been experimentally and theoretically studied for a wide variety of kinematics and geometries, symmetric or asymmetric, coplanar or non-coplanar. See, for example, [1-3] for some reviews. A large body of the published works concerns the ionization of helium. This has resulted in an increasing and encouraging agreement between theoretical predictions and experimental data for simple targets such as $\mathrm{H}$ and $\mathrm{He}$, and has allowed for the exploration of the interaction dynamics in more and more detail.

However, the situation does not look as satisfactory for more complex, many-electron atomic targets (not to mention molecular targets) for which the agreement between experiment and theory deteriorates significantly. This is illustrated for instance by the recent study published by 
Catoire et al [4] on the ionization of helium and argon by fast electrons (see also references quoted in [4]). Catoire et al used so far unexplored kinematics, characterized by large energy transfer and small momentum transfer to the target where the projectile - target interaction is neither glancing, as in the near-dipolar regime, nor a hard knock-on collision, as in the impulsive regime. For He, Catoire et al [4] reported essentially good agreement between experiments and the predictions of two sophisticated theoretical approaches, the hybrid-distorted wave Born approximation coupled with an $R$ matrix (close-coupling) model (DWBA- $R \mathrm{M}$ ) [5] and the Brauner, Briggs and Klar (BBK, often denoted 3C) model [6]. The He experimental results were later confirmed and extended by Stevenson et al [7] who showed in addition an improved agreement of the experimental data with convergent close-coupling (CCC) [8] theoretical predictions. Similar findings were also reported very recently by Dey et al [9] using both the BBK approximation and the Glauber approximation [10]. All of this supports the idea that (e, 2e) triple differential cross-sections for ionization of simple targets such as helium in coplanar geometries can now be predicted very accurately by sophisticated theoretical models, for example (but not solely) the CCC [8] method. In contrast, the study of argon ionization by Catoire et al [4] highlighted some deficiencies in the state-of-the-art theoretical models used for comparison in the case of many-electron atoms, namely the DWBA-RM and the BBK models. In particular, deviations between theory and experiment were reported concerning the recoil intensity distribution, or the relative size of the binary versus recoil peaks, and their angular position. Therefore, it is the aim of this work to further pursue this investigation of 'beyond helium' systems, under similar kinematics characterized by large energy transfer and small momentum transfer to the target, in order to contribute to elucidating the origin of these remaining discrepancies. To this purpose, a joint effort was undertaken between two experimental laboratories (Orsay, France and Adelaide, Australia) and four theoretical groups (in France, USA and Australia) with the objective of providing experimental data measured using two different experimental techniques but under exactly the same kinematical conditions. These data are compared with the results of various sophisticated theoretical approaches currently in use for treating ionization of manyelectron systems, to further guide ongoing theoretical efforts. In this joint study, it was decided to investigate ionization of neon instead of argon studied in [4] since neon is an intermediate case between the 'simple' $\mathrm{He}$ case and the 'complex' argon case, and hence is a good candidate to be a test case for comparing theories with experiments. Also, ionization from both the outer $2 \mathrm{p}$-shell and the inner $2 \mathrm{~s}$-shell of neon are considered, in order to assess the theoretical models when treating s- or p-shell ionization.

\section{Experiment}

\subsection{The Orsay experiments}

The experimental set-up currently in use in Orsay, which combines three high-efficiency, multi-angle toroidal electrostatic energy analysers, has been described in detail elsewhere [11]. The experimental procedure is identical to the one reported in [12]. Briefly, an incident electron beam collides with the gas jet formed at the collision centre. A coplanar geometry is used, where all electrons are observed in the collision plane defined by the incident and scattered momentum vectors $\mathbf{k}_{\mathbf{0}}$ and $\mathbf{k}_{\mathbf{a}}$, respectively. The 'slow' ejected electrons (designated with an index ' $b$ ' for convenience) are multi-angle analysed in a double toroidal analyser, set to the energy $E_{\mathrm{b}}=74 \mathrm{eV}$ and over the angular ranges $\theta_{\mathrm{b}}=20$ $150^{\circ}$ and $210-340^{\circ}$, where $0^{\circ}$ is defined by the incident beam direction. In the off-line analysis, the total $\theta_{b}$-angular range is divided into sectors of width $\Delta \theta_{\mathrm{b}}=5^{\circ}$. The 'fast', forwardscattered electron (indexed ' $a$ ') is collected by the third toroidal analyser [11] set at the scattered energy $E_{\mathrm{a}}=500 \mathrm{eV}$. In the present work, the a-electron is simultaneously observed at two symmetrical angles, $\theta_{\mathrm{a}}=+\left(6^{\circ} \pm 0.25^{\circ}\right)$ and $-\left(6^{\circ} \pm 0.25^{\circ}\right)$ as set by input slits at the entrance to the electrostatic lenses associated with the toroidal analyser. The incident energy $\left(E_{0}\right)$ is consequently adjusted to fulfil the energy conservation requirement for the target under study, $E_{0}=E_{\mathrm{a}}+E_{\mathrm{b}}+\mathrm{IP}$, where IP is its ionization potential $(21.6 \mathrm{eV}$ for $\mathrm{Ne}-2 \mathrm{p}$ and $48.5 \mathrm{eV}$ for $\mathrm{Ne}-2 \mathrm{~s}$ ).

Because of the low coincidence rate, due in particular to the high ejection energy used, the spectrometer was operated at reduced coincidence energy resolution [13], $\Delta E_{\text {coin }} \sim$ $\pm 2.5 \mathrm{eV}$. This figure is small enough to ensure that, in the Ne-2s case, the observed coincidence signal mainly originated from ionizing collisions where the ion is left in its $2 \mathrm{~s} 2 \mathrm{p}^{6}$ state, and that the contribution from the neighbouring satellite structures, $2 s^{2} 2 p^{4} 3 d$, is negligible [14]. Combining all these figures, the momentum transfer resolution amounts to $\Delta K= \pm 0.02$ au while the spread in the momentum transfer direction is about $\Delta \theta_{K}= \pm 1^{\circ}$.

Finally, we note that complementary (e, 2e) experiments were also performed on $\mathrm{He}$ under exactly the same experimental conditions as those used for $\mathrm{Ne}$ (except for a slight change in incident energy, due to the difference in their IP). The He data are similar to those published in [15], though it was preferred here to re-measure them immediately before and after the Ne runs for more consistency on the one hand and for monitoring the good response of the spectrometer on the other hand. The new He set of data (discussed below in figure 1) is found to be in very good agreement with the older one in [15] and in excellent agreement with CCC calculations, thus validating the experimental procedure.

\subsection{The Adelaide experiments}

The apparatus used in Adelaide is a conventional coincidence spectrometer, and full details of the experimental arrangement may be found in [16]. An electron gun, using a tungsten filament as the electron source, produces an incident beam of electrons; the electron beam crosses a target gas beam, at right angles. Two hemispherical electron energy analysers equipped with channel electron multipliers are mounted in the same plane as the electron gun, on rotatable turntables. The two outgoing electrons from the ionization event are detected 


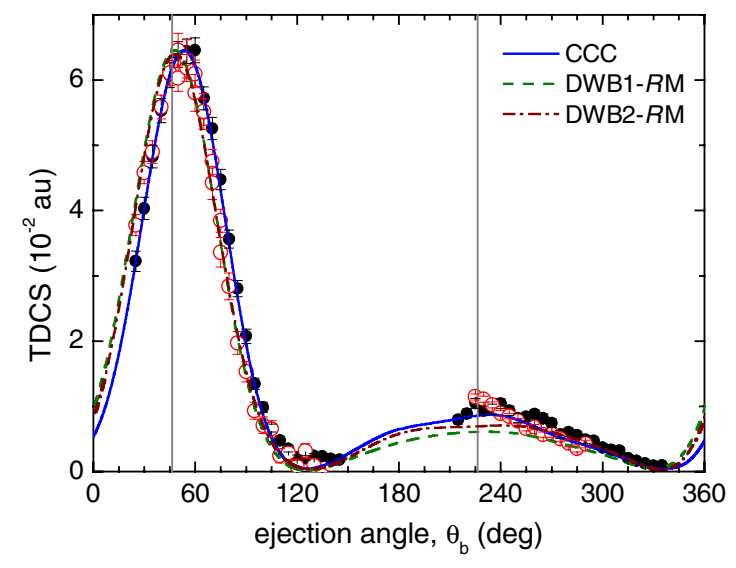

Figure 1. (e, 2e) TDCS for ionization of $\mathrm{He}\left(1 \mathrm{~s}^{2}\right)$, plotted versus ejection angle $\theta_{\mathrm{b}}$, at fixed scattering angle, $\theta_{\mathrm{a}}=-6^{\circ}$. Kinematical parameters are $E_{0}=598.6 \mathrm{eV}, E_{\mathrm{a}}=500 \mathrm{eV}$ and $E_{\mathrm{b}}=74 \mathrm{eV}$. The vertical thin lines indicate the momentum transfer direction, $\theta_{K}$ and its opposite, $\pi+\theta_{K}$. The blue solid line represents the CCC theoretical results. Green dashed and red dash-dotted lines represent the hybrid DWB1-RM and DWB2-RM theoretical results, respectively (practically indistinguishable at the binary lobe). For the sake of clarity, the results of the DWBA-G model are not shown as they are essentially identical to the $\mathrm{CCC}$ results after rescaling by a factor of $\times 2.23$. Solid and open circles: experimental data from Orsay and Adelaide, respectively, with the error bars representing one standard deviation statistical error. When not shown, the error bars are smaller than the size of the circle. The relative experimental data have been normalized for the best visual agreement with theory. The absolute scale shown is that of the CCC calculations. The hybrid DWB1- $R \mathrm{M}$ and DWB2- $R \mathrm{M}$ results have been multiplied by 0.92 .

in the coplanar asymmetric kinematics, with the faster electron being detected at a fixed forward angle of $-6^{\circ}$, while the detection angle of the slow ejected electron is varied in the scattering plane. In contrast to the apparatus used in Orsay, in such 'conventional' coincidence spectrometers the ejected electron can only be detected over a limited angular range due to the physical constraints imposed by the relative positions of the electron gun and electron energy analysers. For example, in the present apparatus the accessible angular regions are 50$135^{\circ}$ for the binary region and $225-290^{\circ}$ for the recoil region. In the measurements presented here, the angular range in the binary region has been extended down to $30^{\circ}$ by the use of a magnetic angle changer [17]. The implementation of such a device in a coincidence spectrometer is described in [18].

The kinematics used in the measurements performed in Adelaide is identical to those used in the measurements performed in Orsay. A few differences in the experimental parameters are noted here: in the Adelaide experiments, the angular acceptance of each of the two electron energy analysers is approximately $\pm 1.5^{\circ}$, and the coincidence energy resolution is around $1.0 \mathrm{eV}$. With these figures, the momentum transfer resolution amounts to $\Delta K= \pm 0.12$ au while the spread in the momentum transfer direction is about $\Delta \theta_{K}= \pm 6.5^{\circ}$.

In addition, the binary and recoil regions of the angular distribution are measured independently by moving the scattered electron analyser from $-6^{\circ}$ to $+6^{\circ}$. The binary to recoil peak ratio is measured in a separate experiment, in which all experimental parameters are held constant, while the scattered electron analyser is moved alternately between positive and negative scattering angles; the coincidence signal is accumulated for a fixed counting time at each scattering angle.

As in the Orsay study, complementary experiments were also performed on a He target under the same experimental conditions as were used for the neon experiments (figure 1). Additionally, He was investigated using the same kinematics as in a previously published joint experimental and theoretical study [7], and the new and earlier results were in excellent agreement.

\section{Theory}

\subsection{The BBK approach}

The BBK approach has been described in detail elsewhere $[6,19]$ and hence need not be repeated here. The basic idea is that the final state is described by a product of three Coulomb waves (hence the alternative name of $3 \mathrm{C}$ ). The main characteristic of the model is that both the post-collisional interaction (PCI) between the three Coulomb particles in the final state and multiple scattering effects are included. The model exhibits the correct asymptotic Coulomb three-body wavefunction for the ejected and scattered electrons in the field of the residual ion. It has been extensively applied with success to the description of (e, 2e) processes in atoms and molecules [20-24] by reducing the complex problem of many electrons to a problem with just one active electron (which will be ejected after the collision). This model describes the residual ion as a proton. Such an approximation is, of course, not generally adequate, but it allows us to apply the BBK model to the complex case of ionizing a many-electron atom such as neon in the present case or argon in [4] or a complex molecule such as water [24]. In particular, it is well known that the recoil peak strongly depends upon the description of the interaction between the slow ejected electron and the residual target. Since the ejected electron is not slow in our case (when compared to the scattered electron), one might expect that our BBK model would yield at least qualitatively correct results, for instance concerning the positions of the binary and recoil lobes maxima.

\subsection{The DWBA approach}

The distorted-wave Born approximation (DWBA) is a very common tool employed in electron impact ionization studies. In this approximation, direct encounter of the projectile with the target is included only once (the first Born approximation). However, the effect of the atomic field on the projectile and the ionic field on the ejected electron is included implicitly via some kind of a distorting potential. In this particular implementation of the DWBA method, the target electronic structure and the distorting potentials are calculated using the numerical Hartree-Fock (HF) method. The target radial orbitals are found by solving a set of self-consistent $\mathrm{HF}$ equations [25]. The distorted waves are calculated in the frozen-core HF potential of the neutral (incident and scattered 
electrons) or singly ionized (ejected electron) target using the computer code of [26]. The numerical implementation of the method is described in detail in [27]. In the DWBA-G version of this model [28], the post-collision interaction is accounted for by multiplying the DWBA cross-section by the so-called Gamow factor (also known as the Coulomb density of states) which represents the normalization constant of the two-body Coulomb function describing the interaction of the two continuum electrons in the final state [6].

\subsection{The hybrid DWBA-R-matrix approach}

The theoretical and computational method for this approach has been outlined in several papers before and hence will not be repeated here. The basic idea is to describe a 'fast' projectile by a distorted wave, but the initial bound state and the (slow) ejected-electron-residual-ion interaction via an $R$-matrix (close-coupling) expansion [29]. Exchange effects between the projectile and the target are neglected in the model, and their inclusion would be non-trivial due to the fundamentally different treatment of the two electrons involved in the process. However, second-order effects in the projectile-target interaction inside the $R$-matrix box (i.e., at short distances) can be accounted for, albeit after some simplifying assumptions [30].

The principal advantage of the hybrid DWBA- $R$-matrix approach is the fact that it is a general method accompanied by a general (non-relativistic) computer code [31]. Hence, it does not rely on either exact (as in atomic hydrogen) or often very specialized (as in helium) descriptions of the initial target bound state or the final ionic states. Instead, multiconfiguration expansions may be employed to describe these states. Furthermore, exchange between the (slow) ejected electron and the target is treated exactly (in a numerical sense), i.e., it is neither neglected nor simplified via a local potential approximation. Finally, channel-coupling effects (again for the slower of the two outgoing electrons, where they are most important) are accounted for as well.

The specific DWBA- $R$-matrix calculations for the present work were performed in the following way: for the helium target, only the $\mathrm{He}^{+}(1 \mathrm{~s})$ state was used in the close-coupling expansion for the $\mathrm{e}-\mathrm{He}^{+}$collision problem. For the rather high ejected-electron energy considered here, this is a very good approximation for this part of the problem, as is known from previous work based upon fully perturbative approaches. The initial bound state was calculated using the same (static-exchange) close-coupling expansion, thereby guaranteeing the orthogonality of the initial bound state and any e- $-\mathrm{He}^{+}$scattering state due to the use of the same Hermitian Hamiltonian for different energies of that subsystem. For the neon target, we started with the two-state approximation proposed by Burke and Taylor [32] for the corresponding photoionization problem. Due to the different selection rules for charged-particle impact ionization, however, we allowed for total orbital angular momenta $L=0-6$ in the e- $\mathrm{Ne}^{+}$scattering state. Multi-configuration expansions were employed for the initial $\left(2 s^{2} 2 p^{6}\right)^{1} S$ bound state as well as for the $\left(2 \mathrm{~s}^{2} 2 \mathrm{p}^{5}\right)^{2} \mathrm{P}$ and $\left(2 \mathrm{~s} 2 \mathrm{p}^{6}\right)^{2} \mathrm{~S}$ states of $\mathrm{Ne}^{+}$. The latter two states were closely coupled to describe the $\mathrm{e}-\mathrm{Ne}^{+}$half-collision problem between the ejected electron and the residual ion.

\subsection{The convergent close-coupling approach}

The convergent close-coupling (CCC) approach to ionization of helium that is relevant to asymmetric kinematics has been detailed by Bray and Fursa [33]. Briefly, the total electronhelium wavefunction is expanded using a set of squareintegrable (eigen and pseudo) states obtained by diagonalizing the target Hamiltonian in two-electron configurations of Laguerre basis functions. The usage of a complete basis ensures that by simply increasing the number of states the expansion converges to the required wavefunction. The original CCC method was intended for dealing with electronimpact excitation of atomic hydrogen [34] and helium [35], but was easily extended to ionizing collisions by associating these processes with excitation of the positive-energy pseudostates [33]. For the currently considered kinematics there are several considerations of interest. The first is that given the highly asymmetric kinematics of the outgoing electrons and high incident energy exchange may be neglected, and convergence in target-space angular momenta is achieved with relatively low $l_{\max }=5$. The number of states taken for each $l$ is $20 l$ leading to a total of 105 states and a maximum of 350 channels. The excited helium states are sufficiently well described assuming the frozen-core approximation, where the inner electron is described by the $1 \mathrm{~s}$ orbital of the $\mathrm{He}^{+}$ion. While this approximation leads to a $0.84 \mathrm{eV}$ error in the ionization energy of the helium ground state, we found that improving the quality of the ground state had no significant effect on the presented results.

Extension of the CCC method to neon is more problematic. We treat $\mathrm{Ne}$ as having a frozen $1 \mathrm{~s}^{2}, 2 \mathrm{p}^{6}$ core with the two 2 s electrons being active. In this way $\mathrm{Ne}$ is treated as being helium-like, which is a rather severe approximation, but allows us to obtain some ionization results for the case where the ion is left in the ${ }^{2} \mathrm{~S}$ state. The core electrons are obtained by solving the self-consistent field Hartree-Fock equations for the ${ }^{2} \mathrm{~S}$ state of the ion. The ionic Hamiltonian is then diagonalized to obtain the Laguerre-based orbitals to construct the two-electron configurations, which are then used to obtain the Ne states upon a diagonalization of the Ne Hamiltonian. Convergence in the presented results is obtained with similar parameters as in the case of helium.

\section{Results and discussion}

The discussion of the results is organized in two parts. First, the angular distributions measured by each group for $\mathrm{He}$ and $\mathrm{Ne}$ are compared with each other and, in the He case, with well-established calculated CCC results. Similarities and differences observed between the two experiments are discussed. Second, the angular distribution of the measured TDCS for both $\mathrm{Ne}-2 \mathrm{p}$ and $\mathrm{Ne}-2 \mathrm{~s}$ orbitals is compared to the theoretical predictions of the various state-of-the-art theoretical models described in the previous section, which allows pin pointing strengths and weaknesses of each model. 


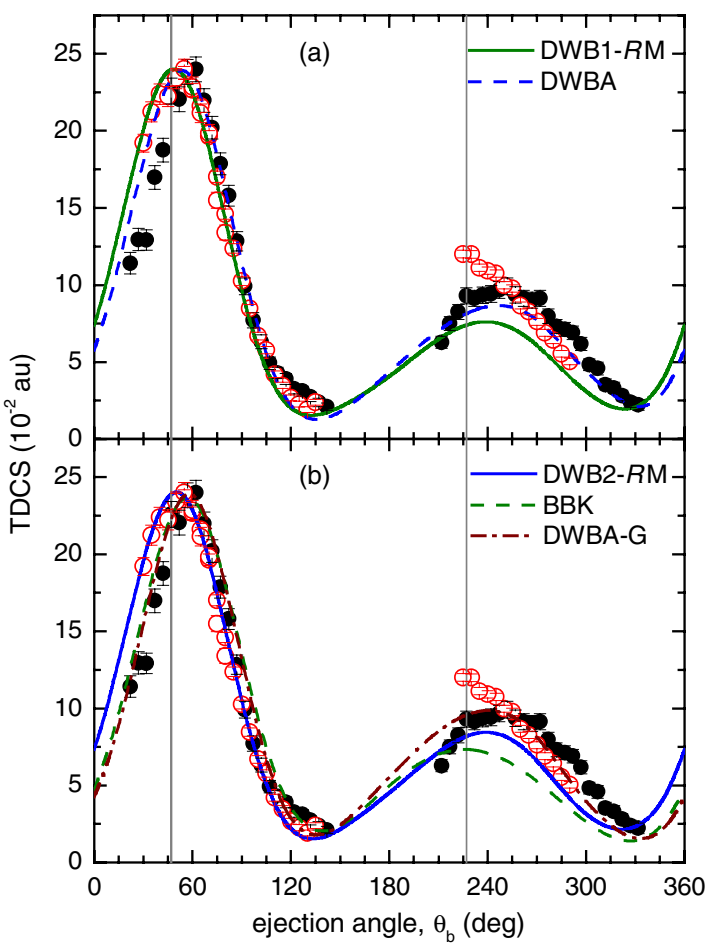

Figure 2. Same as in figure 1, but for ionization of $\mathrm{Ne}\left(2 \mathrm{p}^{6}\right)$, with $E_{0}=595.6 \mathrm{eV}$. Panel (a): green solid and blue dashed lines represent the hybrid DWB1-RM and the DWBA theoretical results, respectively. Panel (b): blue solid, green dashed and red dash-dotted lines represent theoretical results for the hybrid DWB2-RM, the BBK models and the DWBA-G, respectively. The relative experimental data have been normalized for the best visual agreement with theory. The absolute scale shown is that of the hybrid DWB1- $R$ M calculations. The DWBA-G, the hybrid DWB2-RM and the BBK results have been multiplied by $1.73,1.06$ and 1.45 , respectively.

\subsection{Measured angular distributions of the TDCS}

The experimental results obtained both in Orsay and in Adelaide for the TDCS distribution for ionization of $\mathrm{He}$ are shown in figure 1, whereas figures 2 and 3 show the similar results for ionization of $\mathrm{Ne}-2 \mathrm{p}$ and $\mathrm{Ne}-2 \mathrm{~s}$, respectively.

We first comment on the $\mathrm{He}(\mathrm{e}, 2 \mathrm{e})$ results in figure 1 . It is nowadays a well-established fact that at high and intermediate impact energy the ionization process is very well described by the convergent close-coupling (CCC) method in the case of the helium atom. Therefore, the data are compared with calculated CCC results. Note that the relative experimental data from both groups have been independently normalized to the absolute scale given by theory. Remarkably, an almost 'perfect' agreement is observed between the two experimental sets of data, and between the data and CCC theory, both in the shape of the distribution and in the position of the binary and recoil lobes. Additionally, and as pointed out above, $\mathrm{He}$ was investigated in each group using the same kinematics as were used in previously published works [7, 15], and the new and earlier results are in excellent agreement. The CCC results show a shift of the binary lobe of some $8^{\circ}$ from the momentum transfer direction $\left(\theta_{K}\right)$, and so do our data. These observations

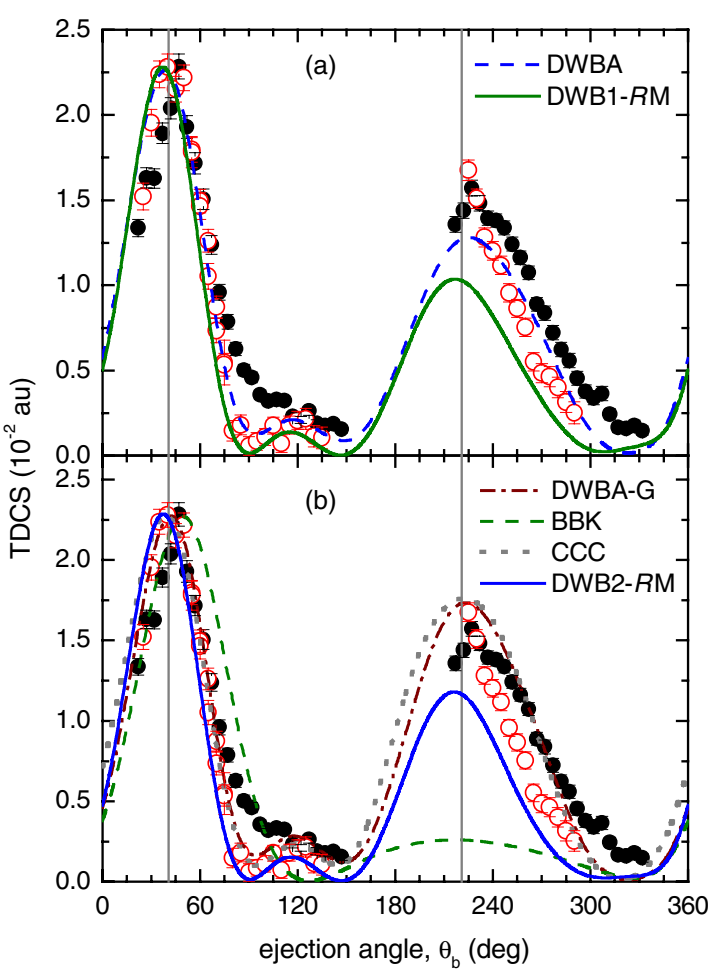

Figure 3. Same as in figure 2, but for ionization of $\mathrm{Ne}\left(2 \mathrm{~s}^{2}\right)$, with $E_{0}=622.5 \mathrm{eV}$. Panel (a): blue dashed and green solid lines represent the DWBA and the hybrid DWB1-RM theoretical results, respectively. Panel (b): red dash-dotted, green dashed, grey dotted and blue solid lines represent theoretical results for the DWBA-G, the $\mathrm{BBK}$, the $\mathrm{CCC}$ and the hybrid DWB2- $R \mathrm{M}$ models, respectively. The relative experimental data have been normalized for the best visual agreement with theory. The absolute scale shown is that of the hybrid DWB1- $R$ M calculations. The DWBA-G, the hybrid DWB2- $R \mathrm{M}$, the BBK and the $\mathrm{CCC}$ results have been multiplied by $1.35,1.01,0.39$ and 0.77 , respectively.

are consistent with known trends for He [1, 2], where peak shifts away from $\theta_{K}$-direction are to be expected whenever the first Born approximation is not sufficiently accurate. We thus believe that the two experiments are free from any significant error or artefact. Our experimental procedures can thus be applied with good confidence to the other target studied here, since the $\mathrm{Ne}$ data were obtained under exactly the same experimental conditions as those used for He.

Also displayed in figure 1 are the results of the hybrid distorted wave first Born $R$-matrix (DWB1- $R \mathrm{M}$ ) model in order to illustrate the degree of agreement with the 'reference' CCC results. For the sake of clarity, the other first-order model considered here, the DWBA, is not represented as it essentially gives results similar to those of the hybrid DWB1-RM model. Indeed, both these models yield practically the same absolute magnitude of the cross-section as the CCC, since they differ from CCC by only about $8 \%$ and $14 \%$, respectively, at the maximum of the binary lobe. Also, both DWBA and DWB1$R \mathrm{M}$ yield no shift of the binary lobe from the $+\mathbf{K}$ direction, and slightly underestimate the recoil intensity. If instead we use the second Born version of the $R$-matrix model, i.e. the hybrid DWB2-RM (also shown in figure 1), these observations are 
only marginally changed (for instance, the maximum recoil intensity is brought closer to the experiments), meaning that second-order effects in the projectile-target interaction at short range (i.e., a second hit of the projectile with any of the target electrons) are small for helium under the considered kinematics. If, on the other hand, we use the version of the DWBA model modified by the Gamow factor, denoted as DWBA-G, we achieve a near-perfect agreement with the reference CCC result as to the shape of the cross-section angular distribution (for this reason it is not shown in figure 1). However, the absolute scale is not correct anymore, though it can be restored via an extra scaling factor of $\times 2.23$. This means that for helium the post-collisional interaction of the two outgoing electrons is significant and accounts for most of the observed difference in the shape of the CCC and DWBA cross-sections.

In the neon case, the comparison between the two experiments is a bit more contrasted. For both $\mathrm{Ne}-2 \mathrm{p}$ and $\mathrm{Ne}-2 \mathrm{~s}$ ionization, figures 2 and 3, respectively, reasonably good general agreement can be observed at both the binary and the recoil lobes, though with some similarities and some differences. Among the similarities, we note that both experiments yield about the same angular positions of the $-2 p$ and -2 s binary lobes, which are consistent with a small (few degrees) shift from the $+\mathbf{K}$ direction. Both measured binary intensity distributions are non-symmetrical with respect to the $+\mathbf{K}$ direction. We also note the same binary-to-recoil intensity ratio in the $-2 \mathrm{~s}$ case. The differences between the two sets of data are twofold: first, a global shift exists in the recoil intensity distributions measured in Orsay, both for $\mathrm{Ne}-2 \mathrm{p}$ and $\mathrm{Ne}-2 \mathrm{~s}$. Indeed, the Adelaide data seem to peak at $-\mathbf{K}$ direction whereas the Orsay data are globally shifted and peak at a larger angle. Second, the Ne-2s (but not 2p) Adelaide data show a minimum at $\theta$ about $90-95^{\circ}$ and a secondary maximum at about $120^{\circ}$, while the Orsay data only show a shoulder in this angular range. The origin of these differences is difficult to ascertain. Both experiments have been carefully checked over and over. We also recall that both experiments are found to be in excellent agreement with each other and with $\mathrm{CCC}$ for He, under the same kinematical conditions as for neon, excluding any angular miscalibration in each of them. A number of avenues have been unsuccessfully explored to try to explain these differences. These are: (i) one may think that the origin of the minimum at $\sim 90^{\circ}$ could be related to the node of the $\mathrm{Ne}-2 \mathrm{~s}$ initial-state momentum space wavefunction. The momentum density $\rho(p)$ indeed has a node at $p$ about 4 au [36]. But this is ruled out, since our $p$-values range here between 1.3 au and 3.4 au. (ii) It might be tempting to blame the lower angular resolution in the Orsay data for the ejected electron channel, $\Delta \theta_{\mathrm{b}}=5^{\circ}$ as compared to approximately $\pm 1.5^{\circ}$ in the Adelaide data. To test this, we convoluted Adelaide's data with a Gaussian function whose FWHM is $5^{\circ}$ : we observe essentially no change, that is, the minimum at $\sim 90^{\circ}$ is not filled in. In fact, one would need to assume a completely unrealistic $45^{\circ}$ width of the Gaussian in order to make the minimum in Adelaide's data 'resemble' the shoulder in Orsay's data. (iii) On the other hand, the angular resolution on the scattered electron channel is better in the Orsay experiments, $\Delta \theta_{\mathrm{a}}= \pm 0.25^{\circ}$, than in the Adelaide experiments, approximately $\pm 1.5^{\circ}$. This translates into a momentum resolution of $\Delta K= \pm 0.02$ au and \pm 0.12 au, and in a spread in the momentum transfer vector direction, $\Delta \theta_{K}= \pm 1^{\circ}$ and $\pm 6.5^{\circ}$ in the Orsay and Adelaide cases, respectively. But we do not see how this would produce a minimum intensity instead of a shoulder. (iv) The coincidence energy resolution of the Orsay experiments $\left(\Delta E_{\mathrm{c}}=2.5 \mathrm{eV}\right)$ is poorer than the Adelaide experiments $\left(\Delta E_{\mathrm{c}}\right.$ around $\left.1.0 \mathrm{eV}\right)$. Nevertheless, it is sufficient to ensure that the measured signal is mainly due to collisions where the ion is left in its ground state, $2 \mathrm{~s} 2 \mathrm{p}^{6}$, and that the contribution from the neighbouring satellite structures, $2 s^{2} 2 p^{4} 3 d$, is negligible [14]. Therefore, it is difficult here again to relate the absence of a minimum in the Orsay data to the modest energy resolution used. Thus the question as to the origin of these differences remains open, and clearly calls for the need for a third, independent experiment to hopefully clarify the situation.

\subsection{Comparison with theoretical models}

The experimental results obtained both in Orsay and in Adelaide for ionization of $\mathrm{Ne}-2 \mathrm{p}$ and $\mathrm{Ne}-2 \mathrm{~s}$ are compared in figures 2 and 3 to the theoretical predictions of the various models considered in this work. For the sake of clarity, the results of the first-order models (in the projectile-target interaction) are grouped in panels (a) whereas those of 'beyond first-order' models are grouped in panels (b). At variance with the He case where the CCC model was taken as a 'reference model' for the absolute scale, it was decided here to rather display the results using the absolute scale of the DWB1-RM model for three reasons: (i) the CCC model is well established for reproducing $\mathrm{H}$ and $\mathrm{He}$ data, while it is here extended for the first time to a heavier target; (ii) the CCC results are only available for the $\mathrm{Ne}-2 \mathrm{~s}$ case but not for the $2 \mathrm{p}$ case; (iii) from figure 1, we note that the absolute scale yielded by the DWB1$R \mathrm{M}$ model for He is quite reliable, being close to that of CCC. The neon experimental data and all other theoretical results are subsequently renormalized to the DWB1-RM ones at the maximum of the binary lobe, as indicated in the figure captions.

Although the experiments are obtained only on a relative scale, it is certainly interesting to note that the various models differ by large factors in the absolute magnitude of the crosssection (up to a factor 3.6 between the smallest and the largest prediction). Briefly, the two first-order models (DWBA and DWB1-RM) yield about the same magnitude (to within less than $10 \%$ ) for the $\mathrm{Ne}-2 \mathrm{p}$ case, while they differ by a factor 0.646 in the -2 s case. The inclusion of the Gamow factor in the DWBA-G model substantially affects the magnitude of the cross-section for both $2 p$ and 2 s cases (though we will see below that it yields a better shape). The $2 \mathrm{~s}$ CCC results are quite close to those of the DWBA. With respect to DWB1-RM, the BBK model yields a too small (too large) binary peak for the $2 p$ and 2 s cases, respectively. Finally, the inclusion of second-order contributions in the projectile-target interactions (DWB2-RM model) affects only very little the magnitude of the cross-section. Given this spread in absolute scale between the various theoretical models, it would certainly 
be desirable to have new experiments where the absolute scale is determined, as done for instance in [37], in order to help distinguishing between the different models. However, this is far from being a trivial issue.

Concerning the shape of the $\mathrm{Ne}-2 \mathrm{p}$ angular distributions, we note from figure 2 that globally speaking, all theories satisfactorily agree with each other as well as with the two experimental sets of data, both for the angular position of the lobes and for the intensity distribution. Looking more in detail in the recoil region where the experiments show some differences (discussed above), we note that (i) both BBK and DWB1-RM underestimate the recoil intensity. (ii) The second Born hybrid DWB2-RM results yield only a slight increase in the recoil intensity, indicating (as in the He case) that second-order effects in the projectile-target interaction at small distances are small under the considered kinematics. (iii) The DWBA is closer to both experiments, though it seems to favour the Orsay one as far as the position of the recoil lobe is concerned, while (iii) the introduction of the Gamow factor in DWBA-G yields good agreement with both experiments, though the agreement is again better with the Orsay data for what concerns the angular position of the recoil lobe.

The situation is more complex for the $\mathrm{Ne}-2 \mathrm{~s}$ angular distributions (figure 3) where substantial differences between the theories are observed. As to the angular position of the binary lobe, the DWB1-RM, the DWB2-RM, the DWBA and the CCC models predict no shift with respect to the $+\mathbf{K}$ directions. In contrast, the DWBA-G model yields a small (few degrees) shift, which is consistent with both experiments, whereas the BBK model predicts a larger shift. In this respect, it is to be noted that both these models include the PCI effects, though in different ways: the BBK accounts for these effects at all distances via the Coulomb wavefunctions, whereas the DWBA-G does take into account the scattered electron-ejected electron interactions (not fully included in the DWBA) via the Gamow factor, i.e. via the normalization factor of the two-body Coulomb function. This stresses the importance of including the PCI to achieve good agreement with experiments. On the other hand, all the considered models except for the BBK are in better agreement with the Adelaide data in the binary region and do produce a secondary maximum at about $120^{\circ}$. For the recoil lobe, the DWB1-RM, the DWB2-RM, the BBK and the CCC produce essentially no shift from the $-\mathbf{K}$ direction, but now the DWBA and to a larger extent the DWBA-G are shifted towards larger angles, in better agreement with Orsay data. The binary-to-recoil intensity ratio observed in both experiments is well described by the CCC and the DWBA$\mathrm{G}$ models, but both these models are closer to the intensity distribution measured at Orsay as to the shape of the recoil lobe. In the DWBA-G case, the good agreement emphasizes again the importance of taking into account the post-collisional interaction. However, this is not the only ingredient that matters. The BBK model fails in describing the recoil intensity distribution although it does account for the PCI. Such failure of the BBK in the case of the ionization of the inner 2s-shell is likely to be attributed to the fact that the presence of the 2 p-electrons is completely neglected in this model. This is different from the case of the ionization of the outer $2 \mathrm{p}$-shell (figure 2), where it seems to be not so important to account for the presence of the 2 s-electrons.

\section{Conclusion}

We have reported new (e, 2e) results for ionization of $\mathrm{He}$ and $\mathrm{Ne}$ under kinematics characterized by large energy transfer and close to minimum momentum transfer from the projectile to the target. The experimental results obtained in two laboratories are used as a sensitive test of a number of state-of-the-art available theoretical models for multielectron atoms. The He results are in excellent agreement with the well-accepted CCC as well as with DWBA-G and hybrid DWB1-RM models, thus validating the experimental procedure. Concerning the $\mathrm{Ne}-2 \mathrm{p}$ data, the various theoretical models used here all give a reasonably good agreement with experiments as to the shape of the angular distributions. However, these models largely differ as to the magnitude of the cross-section; hence the need for measuring absolute cross-sections in future experiments. Concerning the Ne2s data, the situation is more contrasted. The comparison between the two experiments shows two main differences: the first one concerns the existence of either a secondary maximum or a shoulder at about $120^{\circ}$ ejection angle; and the second one is about the existence of an angular shift in the position of the recoil lobe. Only a third independent experiment could clarify these points. Overall, the best general behaviour is obtained for the DWBA-G and the CCC models, which are quasi indistinguishable for what concerns the shape of the distribution. The comparison between theories and experiments demonstrate the importance of including the PCI effects to properly reproduce the lobes positions and intensities. The DWBA-G model takes into account both the electronic structure of the collision system and the effect of the PCI. The BBK approach does account for PCI but ignores completely the electronic structure of the target representing it by a structureless Coulomb centre. The CCC approach can, in principle, describe both the electronic structure and the PCI effects but it is only implemented so far for hydrogen- or helium-like targets, and not for heavier noble gas atoms.

Another important observation is that in all cases ( He and $\mathrm{Ne})$ second-order effects in the projectile-target interaction at short distances are small under the considered kinematics, as demonstrated by the very similar DWB1-RM and DWB2-RM predictions.

\section{Acknowledgments}

AN acknowledges a doctoral grant from the 'Agence Universitaire de la Francophonie' (AUF). KB acknowledges support from the United States National Science Foundation under grant PHY-0244470. MS and BL would like to acknowledge the support of the Australian Research Council.

\section{References}

[1] Ehrhardt H, Jung K, Knoth G and Schlemmer P 1986 Z. Phys. 1 3-32 
[2] Lahmam-Bennani A 1991 J. Phys. B: At. Mol. Opt. Phys. 242401

[3] Weigold E and McCarthy I E 1999 Electron Momentum Spectroscopy (New York: Plenum)

[4] Catoire F, Staicu Casagrande E M, Nekkab M, Dal Cappello C, Bartschat K and Lahmam-Bennani A 2006 J. Phys. B: At. Mol. Opt. Phys. 39 2827-38

[5] Fang Y and Bartschat K 2001 J. Phys. B: At. Mol. Opt. Phys. 34 L19

[6] Brauner M, Briggs J S and Klar H 1989 J. Phys. B: At. Mol. Opt. Phys. 222265

[7] Stevenson M A, Lohmann B, Bray I, Fursa D S V and Stelbovics A T 2007 Phys. Rev. A 75034701

[8] Bray I, Fursa D V, Kheifets A and Stelbovics A T 2002 J. Phys. B: At. Mol. Opt. Phys. 35 R117

[9] Dey R, Roy A C and Dal Cappello C 2008 Nucl. Instrum. Method B 266 242-9

[10] Glauber R J 1959 Lectures in Theoretical Physics vol 1 ed W E Brittin and L G Dunham (New York: Interscience)

[11] Catoire F, Staicu Casagrande E M, Lahmam-Bennani A, Duguet A, Naja A, Ren X G, Lohmann B and Avaldi L 2007 Rev. Sci. Instrum. 78013108

[12] Naja A, Staicu Casagrande E M, Lahmam-Bennani A, Nekkab M, Mezdari F, Joulakian B, Chuluunbaatar O and Madison D H J 2007 J. Phys. B: At. Mol. Opt. Phys. 40 3775-83

[13] Dupré C, Lahmam-Bennani A and Duguet A 1991 Meas. Sci. Technol. 2327

[14] Samardzic O, Braidwood S W, Weigold E and Brunger M J 1993 Phys. Rev. A 484390

[15] Staicu Casagrande E M, Naja A, Mezdari F, Lahmam-Bennani A, Bolognesi P, Joulakian B, Chuluunbaatar O, Al-Hagan O, Madison D H, Fursa D V and Bray I 2008 J. Phys. B: At. Mol. Opt. Phys. 41025204

[16] Haynes M A and Lohmann B 2000 J. Phys. B: At. Mol. Opt. Phys. 334711

[17] Read F H and Channing J M 1996 Rev. Sci. Instrum. 672372
[18] Stevenson M A and Lohmann B 2006 Phys. Rev. A 73 020701(R)

[19] Joulakian B, Dal Cappello C and Brauner M 1992 J. Phys. B: At. Mol. Opt. Phys. 252863

[20] Brauner M, Briggs J S, Klar H, Broad T J, Rösel T, Jung K and Ehrhardt H 1991 J. Phys. B: At. Mol. Opt. Phys. 24657

[21] Berakdar J and Briggs J S 1994 Phys. Rev. Lett. 723799

[22] Zhang S 2000 J. Phys. B: At. Mol. Opt. Phys. 333545

[23] Stia C, Fojon O, Weck P, Hanssen J, Joulakian B and Rivarola R D 2002 Phys. Rev. A 66052709

[24] Champion C, Dal Cappello C, Houamer S and Mansouri A 2006 Phys. Rev. A 73012717

[25] Chernysheva L V, Cherepkov N A and Radojević V 1976 Comput. Phys. Comm. 1157

[26] Chernysheva L V, Cherepkov N A and Radojević V 1979 Comput. Phys. Comm. 1887

[27] Chernysheva L V and Amusia M Ya 1997 Computation of Atomic Processes: A Handbook for the ATOM Programs (Bristol: Institute of Physics Publishing)

[28] Kheifets A 1993 J. Phys. B: At. Mol. Opt. Phys. 262053

[29] Bartschat K and Burke P G 1987 J. Phys. B: At. Mol. Phys. 203191

[30] Reid R H G, Bartschat K and Raeker A 1998 J. Phys. B: At. Mol. Opt. Phys. 31563

Reid R H G, Bartschat K and Raeker A 2000 J. Phys. B 335261 (corrigendum)

[31] Bartschat K 1993 Comput. Phys. Commun. 75219

[32] Burke P G and Taylor K T 1975 J. Phys. B: At. Mol. Phys. 82620

[33] Bray I and Fursa D V 1996 Phys. Rev. A 54 2991-3004

[34] Bray I and Stelbovics A T 1992 Phys. Rev. A 46 6995-7011

[35] Fursa D V and Bray I 1995 Phys. Rev. A 52 1279-98

[36] Lahmam-Bennani A, Duguet A and Dal Cappello C 1986 J. Electr. Spectro. Relat. Phenom. 40 141-61

[37] Daoud A, Lahmam-Bennani A, Duguet A, Dal Cappello C and Tavard C 1985 J. Phys. B: At. Mol. Phys. 18 141-53 